\title{
Influencia de la desconexión moral y el prejuicio sobre la percepción de
} términos asociados a la discapacidad

\section{Influence of moral disengagement and prejudice on the perception of terms associated with disability}

\author{
Jessica Garabal-Barbeira, Pablo Espinosa, Thais Pousada García \\ Universidade da Coruña
}

\begin{abstract}
Resumen
La diversidad de categorías diagnósticas y de calificativos utilizados para hacer referencia a las personas con discapacidad constituyen uno de los problemas más acusados para la inclusión educativa. Utilizando una muestra de 283 estudiantes universitarios, se ha llevado a cabo un estudio correlacional en el que se han recogido sus actitudes, su contacto con la discapacidad y su nivel de desconexión moral. Además, han tenido que evaluar, en función de su grado de negatividad, una serie de términos que se utilizan para referirse a personas con discapacidad, posteriormente agrupados en tres categorías: ofensivos, ambiguos y no ofensivos. Los resultados indican que las variables analizadas predicen la percepción de los términos ambiguos. Además existe una compleja interacción entre estas variables, que actúan como mediadores de otras para predecir la percepción de los términos asociados a la discapacidad.

Palabras clave: discapacidades, educación superior, prejuicio, inclusión, razonamiento moral.
\end{abstract}

\section{Abstract}

The range of diagnostic categories and names used for people with disabilities is one of the most serious problems in Educational Inclusion. We carried out a correlational study with a sample of 283 university students where participants responded to their attitudes, their experience with disabilities and their level of moral disengagement. In addition, they had to evaluate, based on their degree of negativity, a series of terms, used to refer to people with disabilities, subsequently grouped in three categories: offensive, ambiguous and non-offensive. The results show that the variables analyzed in the study predict the perception of ambiguous terms. Additionally, there is a complex interaction between these variables and some act as mediators of others to predict the perception of terms associated with disability.

Keywords: disabilities, higher education, prejudice, inclusion, moral judgment.

\section{Introducción}

La educación inclusiva es un concepto en evolución que trata de ofrecer una educación de calidad a toda la comunidad estudiantil, independientemente de sus condiciones personales o sociales. La historia de la atención a la diversidad ha pasado por diferentes etapas, desde la aceptación de que todos los estudiantes tienen derecho a la educación, hasta ofertar una educación de calidad para todos en las aulas ordinarias (Cedillo, Contreras, Orozco, Hernández, \& Ugalde, 2013).

A lo largo de los años se han utilizado diferentes términos para hacer referencia a las personas con discapacidad, muchos de ellos con connotaciones negativas o que implican un rechazo social. La diversidad de categorías diagnósticas y de calificativos utilizados para hacer referencia a este colectivo constituye uno de los problemas más acusados, con enormes efectos negativos en el área de la inclusión educativa de los estudiantes que padecen algún tipo de discapacidad. Lejos de ser un testimonio de riqueza de matices y de abundancia de posibilidades, el uso de estos términos tan sólo contribuye a dificultar la inclusión real en las aulas (Aguado, Alcedo, \& Flórez, 1997).

Las consecuencias de tal situación son perjudiciales en general, aunque cabe destacar que dificultan, cuando no impiden, la comunicación entre profesionales. Por otro lado, ejercen una influencia negativa directa en las actitudes públicas hacia las personas con discapacidad (Aguado et al., 1997).

Las palabras o términos se asocian a ideas y conceptos que conllevan valores transmitidos en el tiempo. Conscientes de que el lenguaje produce, modifica y orienta el pensamiento, ciertos organismos del ámbito de la salud han intentado acuñar nuevos términos, en busca de una nueva visión social renovada y positiva del colectivo (Romañach \& Lobato, 2005). El objetivo de este estudio es establecer cuáles son las variables cognitivas que podrían determinar la percepción de la comunidad universitaria hacia los términos asociados a las personas con discapacidad.

Se han enunciado las siguientes hipótesis: la frecuencia de contacto con la discapacidad determina la percepción de los términos asociados a dicha condición (h1), pero esta percepción estará mediada, por un lado, por el grado de desconexión moral (h2), por otro, por las actitudes hacia la integración (h3) y por sesgos más específicos, 
como los prejuicios hacia el colectivo de personas con discapacidad (h4).

\section{Método}

\section{Participantes}

La muestra objeto de estudio está compuesta por 283 estudiantes universitarios de la Universidade da Coruña (UDC). De ellos, un $26.1 \%$ son hombres y el $73.9 \%$ son mujeres. La media de edad se sitúa en 24.75 (DT=6.57) años.

\section{Instrumentos}

Para la recogida de datos se ha utilizado una encuesta autoadministrada, distribuida online, compuesta por un conjunto de escalas que se detallan a continuación:

Cuestionario demográfico. (Elaboración propia). Contiene preguntas relevantes sobre el grado de relación y contacto, previo y actual, con personas con discapacidad.

Escala de Valoración de Términos Asociados con la Discapacidad (EVT).

Desarrollada a partir de Aguado et al., (1997). Escala tipo Likert, compuesta por 20 ítems que valoran el grado de negatividad que se le otorga a los términos asociados a la discapacidad del 1-7.

Prejuicios entre los estudiantes hacia las personas con discapacidad. (Suriá, Bueno, \& Rosser Limiñana, 2011). Escala tipo Likert compuesta originariamente por 10 ítems a los que se le han añadido 5 ítems elaborados por los investigadores -para complementar la información recabada con la escala original- evaluables del 1-7 según el grado de acuerdo.

Los ítems añadidos son los siguientes: 11. Las personas con discapacidad, a menudo, recurren a la prostitución para poder mantener relaciones sexuales, 12. A las personas con discapacidad se le facilitan las tareas en el ámbito académico, 13. Las personas con discapacidad suelen estar de mal humor a menudo, 14. Las personas con discapacidad suelen usar su condición para obtener ventajas o beneficios y 15 . Dejaría a mis hijos bajo el cuidado de una persona con enfermedad mental.

Escala de actitudes hacia la discapacidad intelectual. (Cerrillo Martín, Izuzquiza Gasset, \& Egido Gálvez, 2013). Escala tipo Likert compuesta por 22 ítems, en la que se valora el grado de acuerdo del 1-7 con diferentes afirmaciones sobre personas con discapacidad. Se ha suprimido el ítem número 22 por no ser aplicable a la muestra del estudio.

Mecanismos de desconexión moral (MDQ). (Bandura, Barbaranelli, Caprara, \& Pastorelli, 1996). Valora el grado de desvinculación moral a través del grado de acuerdo con diversas afirmaciones del 1-7, compuesta por 32 ítems.

\section{Procedimiento}

Los participantes han cumplimentado una encuesta autoadministrada y anonimizada. El envío de la misma a los encuestados se ha realizado a través del Servicio de Atención a la Diversidad de la Universidade da Coruña.
Se ha llevado a cabo un análisis de fiabilidad de las escalas, un análisis descriptivo y un análisis de mediación a través de un modelo de ecuaciones estructurales.

\section{Resultados}

\section{Análisis de fiabilidad}

El análisis de la fiabilidad de las escalas muestra una consistencia interna aceptable. Los alphas de Cronbach son: $\mathrm{EVT}=.91$, Prejuicios entre los estudiantes hacia las personas con discapacidad $=.84$, Escala de actitudes hacia la discapacidad intelectual $=.88, \mathrm{MDQ}=.86$.

\section{Análisis descriptivo}

A continuación, se comentan las características de la muestra que resultan de interés para el presente estudio.

El $41 \%$ de participantes se encuentran cursando estudios de grado, mientras que únicamente el $3.2 \%$ cursan estudios de doctorado.

El $33.2 \%$ de los participantes pertenecen a la rama de Ciencias Sociales y Jurídicas, el $25.4 \%$ a Ciencias de la Salud, y el $6.7 \%$ pertenecen al área de Artes y Humanidades.

El 70.7\% indican tener algún tipo de contacto previo a la realización de sus estudios universitarios con personas con discapacidad, el $23 \%$ de éstos indican que la razón de este contacto es de carácter familiar y el $24.4 \%$ refieren tenerlo con personas con discapacidad intelectual, siendo este contacto de carácter esporádico en un $34.6 \%$.

Por otra parte, el $53.7 \%$ indican tener algún tipo de contacto actual con personas con discapacidad. La razón de este contacto en un $21.2 \%$ es también de carácter familiar y, en comparación, el 22.3\% refieren tenerlo con personas con discapacidad física, siendo también este contacto de carácter esporádico $18.4 \%$.

Análisis descriptivo de la EVT. (Aguado et al., 1997). Se han agrupado los términos asociados a la discapacidad en tertiles: no ofensivos, ambiguos y ofensivos, en base a los siguientes datos (Tabla 1):

\section{Análisis de datos}


Tabla 1.

Relación de términos asociados a la discapacidad agrupados en subcategorías

\begin{tabular}{|c|c|c|}
\hline & & $M(D T)$ \\
\hline \multirow{6}{*}{$\dot{z} \frac{\sum_{0}^{0}}{\frac{0}{0}}$} & Con diversidad funcional & $2.18(1.54)$ \\
\hline & Con necesidades especiales & $2.62(1.69)$ \\
\hline & Con discapacidad & $2.74(1.79)$ \\
\hline & Excepcional & $3.01(1.97)$ \\
\hline & Discapacitado & $3.75(2.11)$ \\
\hline & Minusválido & $4.00(2.18)$ \\
\hline \multirow{7}{*}{ 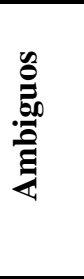 } & Enfermo & $4.39(2.02)$ \\
\hline & Incapacitado & $4.44(2.13)$ \\
\hline & Impedido & $4.96(1.94)$ \\
\hline & Inválido & $5.07(2.01)$ \\
\hline & Disminuido & $5.28(1.79)$ \\
\hline & Inadaptado & $5.67(1.72)$ \\
\hline & Deficiente & $5.79(1.69)$ \\
\hline \multirow{7}{*}{$\frac{\sum_{0}^{0}}{\sum_{0}^{0}}$} & Marginado & $5.94(1.51)$ \\
\hline & Trastornado & $6.06(1.49)$ \\
\hline & Tullido & $6.12(1.45)$ \\
\hline & Anormal & $6.20(1.43)$ \\
\hline & Retrasado & $6.42(1.31)$ \\
\hline & Imbécil & $6.46(1.42)$ \\
\hline & Subnormal & $6.53(1.27)$ \\
\hline
\end{tabular}

La media de los términos no ofensivos se sitúa en la parte baja de la escala $(\mathrm{M}=3.05$; $\mathrm{DT}=1.28)$, por tanto se otorga a estos términos una percepción poco negativa. En cuanto a los términos ambiguos, la media se sitúa en la parte alta $(M=5.08$; DT $=1.45)$, por tanto se otorga a estos términos una percepción bastante negativa. Por último, en referencia a los términos ofensivos, la media se sitúa en la parte alta de la escala $(M=6.24$; $\mathrm{DT}=1.14)$, otorgándole a estos términos una percepción altamente negativa.

En cuanto al grado de negatividad, el $75.3 \%$ de los encuestados considera que el término "retrasado" es altamente negativo y el término percibido como menos negativo de todos los expuestos ha sido "con diversidad funcional", por un $49.8 \%$ de los participantes.

Análisis descriptivo de la escala de Prejuicio. (Suriá et al., 2011). La media se sitúa en la parte inferior de la escala $(M=1.72 ; D T=.63)$, por tanto el grado de prejuicio que muestran es reducido. Únicamente el $21.6 \%$ de los estudiantes indican estar de acuerdo con que las personas con discapacidad no resultan atractivas a otras personas. El 97.9\% están en desacuerdo con que una persona con discapacidad difícilmente puede ser útil.

Análisis descriptivo de la escala de Integración. (Cerrillo Martín et al., 2013). La media se sitúa en la parte alta de la escala $(M=5.84 ; D T=.69)$, por tanto el grado de acuerdo con la integración del colectivo con discapacidad es alto. Los ítems con mayor y menor grado de acuerdo se detallan a continuación: el $27.2 \%$ de los participantes están totalmente de acuerdo con que las personas con discapacidad tienen la misma salud mental que otras personas. El 20.8\% de los encuestados están totalmente de acuerdo con que este colectivo se integra en la sociedad del mismo modo que el resto de la población y, únicamente un $3.9 \%$ están totalmente de acuerdo con que están integrados realmente.

Análisis descriptivo de la escala MDQ. (Bandura et al., 1996). La media se sitúa en la parte inferior de la escala $(M=1.82 ; D T=.56)$, la frecuencia de utilización de sesgos de desconexión moral en los participantes es baja. En cuanto al mayor o menor grado de acuerdo, destacan los siguientes ítems: "los chicos/as que tienen malas condiciones de vida no son culpables de comportarse agresivamente", un $25.1 \%$ indican estar de acuerdo. El $90.8 \%$ no están nada de acuerdo con la afirmación "meterse con alguien realmente no le va a causar daño".

\section{Análisis de mediación}

En base a la tipología de términos asociados a la discapacidad -ofensivos, ambiguos y no ofensivos- se han establecido diferentes relaciones con el grado de desconexión moral, el prejuicio, las actitudes ante la integración y la frecuencia de contacto con personas con discapacidad.

El modelo de ecuaciones estructurales desarrollado explica las relaciones entre las variables de interés para el estudio, así como la predicción de los términos ambiguos mediante estas (Figura 1).

Dicho modelo muestra que las actitudes hacia la integración median la relación entre la frecuencia de contacto y la percepción negativa de los términos ambiguos. Mientras que existe un efecto total significativo entre ambas variables, el efecto directo de frecuencia de contacto deja de ser significativo cuando introducimos las actitudes hacia la integración como mediador.

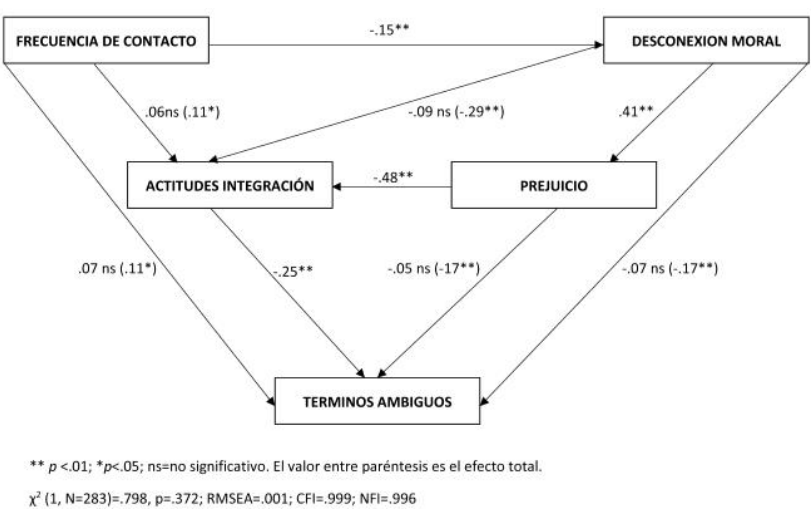

Figura 1. Modelo de ecuaciones estructurales para la percepción de los términos ambiguos mediante la frecuencia de contacto, el grado de desconexión moral, las actitudes hacia la integración y el prejuicio.

A su vez, estas actitudes presentan una relación significativa con la frecuencia de contacto y percepción de los términos, cumpliéndose los criterios de mediación. De este modo, las actitudes hacia la integración se configuran como una variable relevante para explicar la relación entre la frecuencia de contacto y la percepción de los términos.

Por otro lado, la desconexión moral actúa como mediador de la relación entre frecuencia de contacto y 
actitudes hacia la integración. Observamos que el efecto total de la frecuencia de contacto sobre la actitudes hacia la integración es significativo $(.11, \mathrm{p}<.05)$, mientras que el efecto directo, cuando tenemos en cuenta la desconexión moral como variable mediadora, deja de serlo.

El prejuicio actúa como mediador entre la desconexión moral y las actitudes de integración, y de la relación entre la desconexión moral y la percepción negativa de los términos. Por último, las actitudes de integración actúan como mediador entre prejuicio y percepción negativa de los términos ambiguos.

Todo ello muestra una relación compleja entre las variables del estudio donde unas variables median y explican las relaciones existentes entre otras del modelo, a diferentes niveles.

Tabla 2

Correlaciones de las variables del modelo de ecuaciones estructurales

\begin{tabular}{|c|c|c|c|c|}
\hline & 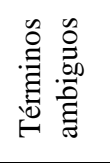 & 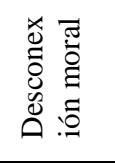 & $\begin{array}{l}\frac{0}{0} \\
\frac{\sqrt[0]{0}}{0} \\
0\end{array}$ & 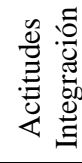 \\
\hline $\begin{array}{l}\text { Términos } \\
\text { ambiguos }\end{array}$ & & & & \\
\hline $\begin{array}{l}\text { Desconexión } \\
\text { moral }\end{array}$ & $-.181 * *$ & & & \\
\hline Prejuicio & $-.220 * *$ & $.413 * *$ & & \\
\hline $\begin{array}{l}\text { Actitudes } \\
\text { Integración }\end{array}$ & $.311 * *$ & $-.301 * *$ & $-.525 * *$ & \\
\hline $\begin{array}{l}\text { Frecuencia de } \\
\text { contacto }\end{array}$ & $.120 *$ & $-.152 *$ & -.111 & $.132 *$ \\
\hline
\end{tabular}

${ }^{*} p<.05, * * p<.01$

Posteriormente, se relacionan entre sí las variables de interés para el propio estudio (Tabla 2), comprobando que todas las variables del modelo correlacionan entre sí salvo prejuicio y frecuencia de contacto.

\section{Discusión}

El lenguaje tiene una influencia trascendental en nuestra percepción de la realidad, condiciona nuestro pensamiento y el de quienes nos escuchan (Balas Lara, 2007). En la mayoría de los casos, la discriminación nace de la palabra. Por ello, este estudio ha pretendido establecer cuáles son las variables cognitivas que podrían determinar la percepción de la comunidad universitaria hacia los términos asociados a las personas con discapacidad.

Según los resultados obtenidos, podemos confirmar las hipótesis establecidas para la investigación: la frecuencia de contacto determina la percepción de los términos asociados a la discapacidad (h1), pero esta percepción estará mediada, por un lado, por el grado de desconexión moral (h2), por otro, por las actitudes hacia la integración (h3) y por sesgos más específicos, como los prejuicios hacia el colectivo de personas con discapacidad (h4). Los resultados muestran que estas relaciones no son tan directas como cabría esperar y que el modelo desarrollado muestra interacciones complejas entre variables.

Sobre la base de una muestra de 283 estudiantes universitarios se ha detectado que el $70 \%$ de éstos han tenido algún tipo de contacto previo a la realización de sus estudios con personas con discapacidad y el 50\% mantienen este contacto en la actualidad. El tipo de contacto más frecuente es con familiares, y la frecuencia de este contacto tiene carácter esporádico.

Nuestros datos sobre las características de este contacto contrastan con los del estudio de Rodríguez-Martín y Álvarez- Arregui (2014). Mientras en su estudio indican que el contacto más común es con personas con discapacidad visual $(58.3 \%)$ y física $(47.9 \%)$ en segundo lugar, en nuestro estudio la categoría más habitual fue discapacidad física $(22.3 \%)$ y con una frecuencia mucho menor.

Los resultados obtenidos se relacionan con los de investigaciones previas, y ayudan a clarificar las relaciones entre las variables analizadas en los casos en los que existe controversia. Por ejemplo, en diversos estudios se ha analizado si el contacto con personas con discapacidad favorece la existencia de actitudes favorables a la inclusión. En el estudio de Gómez e Infante (2004) han detectado que variables como la edad, la existencia o no de contacto, la razón y la frecuencia de dicho contacto o el tipo de grupo con que se tiene contacto, no afectan directamente a las actitudes hacia personas con discapacidad. Flórez García (1999), Nowicki (2006) y Yuker (1994) indican que el conocimiento y la experiencia previas sobre cuestiones relacionadas con la discapacidad, el hecho de ser hombre o mujer, o el tener o no compañeros con discapacidad dentro de la misma aula, no resultan por sí solas variables determinantes, ya que no generan diferencias en las actitudes. Sin embargo, en el estudio realizado por Aguilar, Fernández y Provecho (2003), se encuentra una relación significativa entre el contacto y la tendencia a presentar actitudes más positivas hacia ellos, lo que se encuentra en la línea de nuestra primera hipótesis: "la frecuencia de contacto determina la percepción de los términos asociados a la discapacidad".

Por otro lado, y en relación con la terminología asociada a la discapacidad, han sido pocos los estudios que han indagado sobre el tema. Sin embargo, los autores de la escala EVT, Aguado, Alcedo y Flórez (1997), en su aplicación, obtuvieron entre sus resultados que el término "excepcional" es considerado como el menos negativo, seguido de "enfermo" y de sujeto "con necesidades especiales". Estos datos no son concordantes con los del presente estudio, posiblemente porque la investigación mencionada se realizó en el año 1997, y el uso de conceptos asociados a la discapacidad se ha visto modificado por diversas perspectivas o paradigmas recientes. En la actualidad, el término considerado como menos negativo es "persona con diversidad funcional", seguido de "con necesidades especiales" y "con discapacidad".

Los resultados obtenidos por Aguado et al., (1997) ponen de manifiesto diferencias semánticas entre términos, es decir, unos son valorados menos peyorativamente que otros. Concluyen que los términos 
que denotan las limitaciones o restricciones de las personas con discapacidad a la hora de realizar una determinada actividad, presentan una connotación menos peyorativa $y$, por tanto, permiten predecir una incidencia no negativa, o al menos neutra, en las actitudes hacia las personas con discapacidad. Por el contrario, otros términos son valorados de manera más peyorativa, lo que lleva a suponer que repercutirán negativamente en las actitudes públicas hacia este colectivo.

En un estudio similar de Benitez y Muñoz Pinto (2013) se pudo evidenciar que algunas palabras como "retrasado", "trastornado", "incapacitado", "deficiente" y "discapacitado" causaban mayor malestar que otras, en los participantes por las connotaciones negativas que les rodean. Estos datos corroboran la hipótesis 2: "la percepción de los términos está mediada por el grado de desconexión moral".

Conceptos como "retrasado" y "trastornado" se utilizan asociando la discapacidad física con enfermedad mental. Así mismo los términos "incapacitado", "desvalido" y "deficiente" se relacionan con la discapacidad en general, pues se considera que la persona en esta situación no puede valerse por sí mismo, sino que necesita de otros. La mayoría de los sujetos están de acuerdo en que el término "discapacitado" es el apropiado para denominar a este colectivo. Sin embargo, en el presente estudio el término seleccionado como más apropiado es el de "persona con diversidad funcional".

En el trabajo de Aguado, Alcedo y Arias (2008) se implementó un programa de cambio de actitudes, a cuyo término los participantes presentaron una valoración más positiva de los conceptos asociados con la discapacidad incluidos en la escala EVT que los participantes del grupo control. La eficacia de este programa se ha constatado y los efectos se mantienen hasta tres años después. Este apunte es congruente con nuestra tercera hipótesis: "la percepción de los términos está mediada por las actitudes hacia la integración".

En otro estudio del mismo equipo, Aguado, Flórez y Alcedo (2003), realizado con escolares de $1^{\circ}$ y $2^{\circ}$ de la ESO, se indica que se puede conseguir un cambio positivo en la valoración de términos referentes a personas con discapacidad y, por tanto, las actitudes y el grado de prejuicio hacia ellas. Estos resultados acerca del efecto del cambio en los prejuicios sobre la valoración de términos, da cierto apoyo a nuestra cuarta hipótesis: "la percepción de términos está mediada por los prejuicios hacia el colectivo de personas con discapacidad".

Los resultados obtenidos por Hodge, Davis, Woodard y Sherrill (2002); Krahé y Altwasser (2006); y Slininger, Sherrill y Jankowski (2000) demuestran, por un lado, que las actitudes se pueden cambiar, pero para ello es necesario programar y valorar el cambio y su estabilidad. Por otro, que las técnicas combinadas de contacto estructurado con personas con discapacidad e información resultan eficaces en el cambio de actitudes hacia este colectivo, en la medida en que estas actitudes mejoran y se vuelven más positivas (Aguado et al., 2008).

\section{Conclusiones}

Los resultados obtenidos muestran que los términos ambiguos hacia personas con discapacidad, y por tanto sus significados asociados, están mediados por diversos factores, como la frecuencia de contacto con esta población -previo y actual-, el grado de desconexión moral, las actitudes hacia la integración y el prejuicio hacia este colectivo.

En este sentido, es necesario fomentar acciones de sensibilización que favorezcan el contacto y conocimiento de la realidad del concepto de la discapacidad, y sus connotaciones, para alcanzar su inclusión real en la sociedad y, especialmente, en el ámbito de la educación superior.

\section{Referencias}

Aguado, A., Alcedo, M., \& Arias, B. (2008). Cambio de actitudes hacia la discapacidad con escolares de primaria. Psicothema, 20(4), 697-704.

Aguado, A., Alcedo, M., \& Flórez, M. (1997). Una escala de valoración de términos asociados con discapacidad: primeros resultados. REMA Revista electrónica de metodología aplicada, 2(1), 65-81.

Aguado, A., Flórez, M., \& Alcedo, M. (2003). Un programa de cambio de actitudes hacia personas con discapacidad en entorno escolar. Análisis y modificación de conducta, 29(127), 673-704.

Aguilar, M., Fernández, E., \& Provecho, M. (2003). ¿Tienen los futuros maestros una actitud especialmente favorable hacia la diversidad? Encuentros en Psicología Social, 329-333.

Balas Lara, M. (2007). El tratamiento de la discapacidad. Comunicación y discapacidades, ISBN 978-8, 315320.

Bandura, A., Barbaranelli, C., Caprara, G. V., \& Pastorelli, C. (1996). Mechanisms of moral disengagement in the exercise of moral agency. Journal of personality and social psychology, 71(2), 364.

Benitez, L., \& Muñoz Pinto, C. (2013). Evaluación del impacto de un programa de resignificación de creencias y actitudes en personas con discapacidad y familiares/cuidadores usuarios del Hospital Universitario de Santander. Universidad Pontificia Bolivariana. Recuperado a partir de https://repository.upb.edu.co/handle/20.500.11912/56 5

Cedillo, I., Contreras, S., Orozco, C., Hernández, K., \& Ugalde, D. (2013). Terminología internacional sobre la educación inclusiva. Revista Actualidades Investigativas en Educación, 13(1), 1-29.

Cerrillo Martín, R., Izuzquiza Gasset, D., \& Egido Gálvez, I. (2013). Inclusión de jóvenes con discapacidad intelectual en la Universidad. Revista de Investigación en Educación, 11(1), 41-57.

Flórez García, M. (1999, octubre 5). Actitudes hacia las personas con discapacidad: valoración de la eficacia de un programa de cambio de actitudes en entorno escolar. Universidad de Oviedo. Recuperado a partir de http://digibuo.uniovi.es/dspace/handle/10651/15954

Gómez, V., \& Infante, M. (2004). Actitudes de los estudiantes de educación hacia la integración de 
personas con discapacidad y hacia la educación multicultural. Cultura y educación, 16(4), 371-383.

Hodge, S., Davis, R., Woodard, R., \& Sherrill, C. (2002). Comparison of practicum types in changing preservice teachers' attitudes and perceived competence. Adapted Physical Activity Quarterly, 19(2), 155-171.

Krahé, B., \& Altwasser, C. (2006). Changing negative attitudes towards persons with physical disabilities: An experimental intervention. Journal of Community \& Applied Social, 16(1), 59-69. https://doi.org/10.1002/casp.849

Nowicki, E. A. (2006). A cross-sectional multivariate analysis of children's attitudes towards disabilities. Journal of Intellectual Disability Research, 50(5), 335348. https://doi.org/10.1111/j.13652788.2005.00781.x

Rodríguez-Martín, A., \& Álvarez- Arregui, E. (2014). Estudiantes con discapacidad en la Universidad. Un estudio sobre su inclusión. Revista Complutense de Educación, 25(2), 457-479.

Romañach, J., \& Lobato, M. (2005). Diversidad funcional, nuevo término para la lucha por la dignidad en la diversidad del ser humano. Foro de Vida Independiente.

Slininger, D., Sherrill, C., \& Jankowski, C. (2000). Children's attitudes toward peers with severe disabilities: Revisiting contact theory. Adapted Physical Activity Quarterly, 17(2), 179-196.

Suriá, R., Bueno, A., \& Rosser Limiñana, A. (2011). Prejuicios entre los estudiantes hacia las personas con discapacidad: reflexiones a partir del caso de la Universidad de Alicante. Alternativas. Cuadernos de Trabajo Social, 18, 75-90.

Yuker, H. (1994). Variables that influence attitudes toward persons with disabilities: Conclusions from the data. Journal of Social Behavior and Personality, 9(5), 3. 